\title{
Circulating nucleosomes as epigenetic biomarkers in pancreatic cancer
}

\author{
Monika Bauden ${ }^{1}$, Dorian Pamart ${ }^{2}$, Daniel Ansari ${ }^{1}$, Marielle Herzog ${ }^{2}$, Mark Eccleston², Jake Micallef ${ }^{2}$, \\ Bodil Andersson ${ }^{1}$ and Roland Andersson ${ }^{1 *}$
}

\begin{abstract}
Background: To improve the prognosis of patients with pancreatic cancer, new biomarkers are required for earlier, pre-symptomatic diagnosis. Epigenetic mutations take place at the earliest stages of tumorigenesis and therefore offer new approaches for detecting and diagnosing disease. Nucleosomes are the repeating subunits of DNA and histone proteins that constitute human chromatin. Because of their release into the circulation, intact nucleosome levels in serum or plasma can serve as diagnostic disease biomarkers, and elevated levels have been reported in various cancers. However, quantifying nucleosomes in the circulation for cancer detection has been challenging due to nonspecific elevation in sera of patients with benign diseases. Here, we report for the first time differential, disease-associated epigenetic profiles of intact cell-free nucleosomes (cfnucleosomes) containing specific DNA and histone modifications as well as histone variants circulating in the blood. The study comprised serum samples from 59 individuals, including 25 patients with resectable pancreatic cancer, 10 patients with benign pancreatic disease, and 24 healthy individuals using Nucleosomics, a novel ELISA method.

Results: Multivariate analysis defined a panel of five serum cfnucleosome biomarkers that gave an area under the curve (AUC) of 0.95 for the discrimination of pancreatic cancer from healthy controls, which was superior to the diagnostic performance of the common pancreatic tumor biomarker, carbohydrate antigen 19-9 (CA 19-9) with an AUC of 0.87. Combining CA 19-9 with a panel of four cfnucleosome biomarkers gave an AUC of 0.98 with an overall sensitivity of $92 \%$ at $90 \%$ specificity.
\end{abstract}

Conclusions: The present study suggests that global epigenetic profiling of cfnucleosomes in serum using a simple $\mathrm{NuQ}^{\circ}$ immunoassay-based approach can provide novel diagnostic biomarkers in pancreatic cancer.

Keywords: Nucleosomes, DNA, Pancreatic cancer, Epigenetics, NuQ assays, Serum, Diagnosis, Screening

\section{Background}

Pancreatic cancer has a 5-year survival rate of only $6 \%$ [1]. The poor prognosis is mainly due to the asymptomatic nature of its early stages, its aggressive biological behavior, and limitations of current detection technologies. More than $80 \%$ of the patients are inoperable at the time of diagnosis. At present, the diagnosis of small, early-stage tumors that can be surgically resected offers patients the best chances for survival and can increase 5-year survival rates up to $30-40 \%$ [2].

The standard serum marker for pancreatic cancer is carbohydrate antigen 19-9 (CA 19-9). CA 19-9 is a

\footnotetext{
* Correspondence: Roland.Andersson@med.lu.se

'Department of Surgery, Clinical Sciences, Lund, Lund University and Skåne University Hospital, Lund, SE-221 85 Lund, Sweden

Full list of author information is available at the end of the article
}

modified Lewis (a) blood group antigen. The sensitivity of CA 19-9 for the diagnosis of pancreatic cancer is reported as $79 \%$ while the median specificity is $82 \%$ [3]. According to the European Group on Tumor Markers (EGTM) status report, CA 19-9 cannot be recommended for screening purposes but only for monitoring response to treatment in patients who had elevated levels prior to treatment [4]. Therefore, there is an urgent need for new and effective serum markers for the disease.

Apart from classical pancreatic cancer-associated signaling pathways and genetic mutations [5], cancer cells are also subject to epigenetic misregulation including DNA methylation-mediated gene silencing and posttranslational modifications of histone proteins for dynamic chromatin structural regulation [6]. The influences of these processes on the regulation of gene 
expression implicated in pancreatic cancer and opportunities for next-generation treatment were recently reviewed [7]. Epigenetic alterations occur very early in the transformation process, and these changes have been proposed as biomarkers of transformation [8]. In addition to gene-specific epigenetic markers, global levels of epigenetic modifications also provide diagnostic and prognostic information [9]. The importance of epigenetic markers, including histone $\mathrm{H} 3$-specific posttranslational modifications, as prognostic factor in pancreatic cancer has been highlighted recently [10, 11]. Indeed, tumor-specific post-translational modifications of histones influencing gene expression have been identified in biopsy material, and the term "histone onco-modifications" has been proposed for histone modifications linked to cancer [8]. The blood of cancer patients contains cell-free DNA (cfDNA). While the origins of cell-free DNA is subject to debate [12], Mouliere et al. demonstrated that cfDNA in the blood of cancer patients consists of small fragments centered around $166 \mathrm{bp}$ [13]. This is consistent in size with nucleosomal DNA (146 bp) and 20 bp linker DNA protected as circulating cell-free nucleosomes (cfnucleosomes).

Mono- and oligonucleosomes are released by chromatin fragmentation during cell death. As a result, nucleosomes are present in a range of diseases including inflammation, infection, and benign diseases as well as cancer. As such, the reported potential utility of circulating nucleosome quantification has been limited to monitoring therapy efficacy, including radio- and chemotherapy in pancreatic cancer $[14,15]$ and relapse monitoring. However, circulating cfnucleosome measurements have not been used routinely in the clinic as it has not been previously possible to detect tumor-specific, quantitative changes to circulating cfnucleosome levels. Recently developed innovative analytical techniques enabled detection of cfnucleosomes containing histone and DNA modifications as well as histone variants associated with tumor-specific epigenetic changes, not only at the tumor site, but also in the circulation [16-19].

We suggest that quantification of cancer-associated alterations in cell-free nucleosome-bound histone and DNA modifications as well as histone variants could be attractive to investigate as a diagnostic biomarker for early detection of pancreatic cancer.

We report for the first time the diagnostic potential of selected epigenetic profiles from circulating cfnucleosomes in pancreatic cancer using a simple immunoassay profiling platform-Nucleosomics (VolitionRx). In this study, we examine and compare the specificity and sensitivity of the cfnucleosome biomarkers and CA 19-9 serum marker to distinguish pancreatic ductal adenocarcinoma from benign pancreas disease and healthy controls.

\section{Results}

\section{Study design}

This prospective study consisted of 59 individuals and comprised serum samples from patients with pancreatic cancer $(n=25)$, benign pancreatic disease $(n=10)$, and healthy controls $(n=24)$. As detection of late-stage pancreatic cancer is of little clinical value, all subjects included in this study were selected from operable, early-stage disease. All patients underwent pancreatic resection with curative intent, with 23 patients undergoing pancreaticoduodenectomy and 2 patients undergoing distal pancreatectomy. Histological differentiation included well-differentiated in 1 patient, moderately differentiated in 12 patients, and poorly differentiated in 12 patients. Median tumor size was $3.2 \mathrm{~cm}(0.3-8 \mathrm{~cm})$. Additional patient data are provided in Table 1.

\section{Epigenetic profiling of circulating cfnucleosomes using nucleosome assays}

Epigenetic profiles of circulating cfnucleosomes of subjects with pancreatic cancer, subjects with other pancreatic conditions, and healthy control subjects were investigated using ELISA-based $\mathrm{NuQ}^{\circ}$ assays. Nine epigenetic features of serum cell-free nucleosomes were measured, including nucleosome-associated methylated DNA (5-methylcytosine), histone modifications H2AK119Ub, H3K4Me2, H3K9Me3, H3K27Me3, H3K9Ac, and H4Pan-acetylation as well as histone sequence variants $\mathrm{H} 2 \mathrm{AZ}$ and $\mathrm{mH} 2 \mathrm{~A} 1.1$, using a novel, global epigenetic immunoassay approach. The receiver operator characteristic (ROC) curves for

Table 1 Demographics of the study group

\begin{tabular}{|c|c|c|c|c|}
\hline Diagnosis & $\begin{array}{l}\text { No. of } \\
\text { patients }\end{array}$ & $\begin{array}{l}\text { Median } \\
\text { CA 19-9 level } \\
\text { (range) }\end{array}$ & $\begin{array}{l}\text { Median } \\
\text { age } \\
\text { (range) }\end{array}$ & $\begin{array}{l}\text { Male/ } \\
\text { female }\end{array}$ \\
\hline Pancreatic cancer & 25 & \multirow{5}{*}{$\begin{array}{l}150 \mathrm{kU} / \mathrm{l} \\
(1.7-1494 \mathrm{kU} / \mathrm{l})\end{array}$} & \multirow{5}{*}{$\begin{array}{l}69 \\
(46-78)\end{array}$} & \multirow[t]{5}{*}{$15: 10$} \\
\hline $\begin{array}{l}\text { Lymph node } \\
\text { involvement }\end{array}$ & 19 & & & \\
\hline $\begin{array}{l}\text { No lymph node } \\
\text { involvement }\end{array}$ & 6 & & & \\
\hline Stage IIA & 3 & & & \\
\hline Stage IIB & 22 & & & \\
\hline Benign disease & 10 & \multirow{6}{*}{$\begin{array}{l}31 \mathrm{kU} / \mathrm{l} \\
(0.6-300 \mathrm{kU} / \mathrm{l})\end{array}$} & \multirow{6}{*}{$\begin{array}{l}72 \\
(58-77)\end{array}$} & \multirow[t]{6}{*}{$5: 5$} \\
\hline Chronic pancreatitis & 4 & & & \\
\hline $\begin{array}{l}\text { Intraductal papillary } \\
\text { mucinous neoplasms } \\
\text { (IPMN) }\end{array}$ & 2 & & & \\
\hline Serous cystadenoma & 2 & & & \\
\hline $\begin{array}{l}\text { Tubular adenoma in the } \\
\text { ampulla of Vater }\end{array}$ & 1 & & & \\
\hline Benign biliary stricture & 1 & & & \\
\hline Healthy & 24 & $\begin{array}{l}7.3 \mathrm{kU} / \mathrm{I} \\
(4-20 \mathrm{kU} / \mathrm{l})\end{array}$ & $\begin{array}{l}58 \\
(48-70)\end{array}$ & $15: 9$ \\
\hline
\end{tabular}


each nucleosome assay in cancer vs. healthy or benign and cancer vs. healthy groups are provided in Additional file 1 . The area under the curve (AUC) for the individual ROC curves varied from 0.52 to 0.77 for cancer vs. healthy and benign and 0.53-0.81 for cancer vs. healthy (Table 2).

Diagnostic sensitivity for individual nucleosome-based biomarkers (at $90 \%$ specificity) ranged from 0 to $40 \%$ for cancer vs. healthy and benign and from 0 to $60 \%$ for cancer vs. healthy (Table 2).

\section{Multivariate analysis}

The cumulative performance of cfnucleosome biomarkers alone and in combination with CA 19-9 was evaluated using multivariate analysis, optimized for AUC, for discrimination of cancer vs. healthy and benign groups. Linear models, based on a weighted sum of one to five variables (panel size limited to five to avoid overtraining) were developed using Fisher's linear discriminant (LDA) and confirmed by logistic regression (LR) [20] (see the "Methods" section below).

Model 1: $\quad-0.825 \quad(5 \mathrm{MC})-2.909 \quad(\mathrm{H} 2 \mathrm{AZ})+2.641$

(H2A1.1) - $1.050\left(\mathrm{H}^{2} 4 \mathrm{Me}_{2}\right)-0.551$ (H2AK119Ub)

Model 2: $\quad-0.788 \quad(5 \mathrm{MC})-2.338 \quad(\mathrm{H} 2 \mathrm{AZ})+1.959$

$(\mathrm{H} 2 \mathrm{~A} 1.1)+0.672\left(\mathrm{H} 3 \mathrm{~K} 4 \mathrm{Me}_{2}\right)+0.782(\mathrm{CA} 19-9)$

A box plot derived from the optimal panel of five assays (model 1) is shown in Fig. 1. The AUC for discrimination of cancer vs. healthy and benign was 0.92 , which exceeded that of CA 19-9 with an AUC of 0.84 in our cohort (Fig. 2). A box plot for a similar model (model 2), in which the lowest weighted assay (nucleosome-associated H3K119Ub) in model 1 was replaced with CA 19-9, is shown in Fig. 3. The AUC for discrimination of cancer vs. healthy and benign groups increased to 0.94 (Fig. 2). For discrimination of cancer vs. healthy groups, the five cfnucleosome biomarker panel (model 1) had an AUC of 0.95 compared to 0.87 for CA 19-9. The four

Table 2 Nucleosome epigenetic feature, AUC, and sensitivity at $90 \%$ specificity

\begin{tabular}{|c|c|c|c|c|}
\hline \multirow[t]{2}{*}{$\mathrm{NuQ}^{\circledast}$ assay } & \multicolumn{2}{|c|}{$\begin{array}{l}\text { Cancer vs. healthy and } \\
\text { benign }\end{array}$} & \multicolumn{2}{|c|}{ Cancer vs. healthy } \\
\hline & $\mathrm{AUC}$ & Sensitivity (\%) & $\mathrm{AUC}$ & Sensitivity (\%) \\
\hline $\mathrm{H} 3 \mathrm{~K}_{4} \mathrm{Me}_{2}$ & 0.52 & 0 & 0.53 & 0 \\
\hline $\mathrm{mH} 2 \mathrm{~A} 1.1$ & 0.58 & 16 & 0.64 & 40 \\
\hline $\mathrm{H} 3 \mathrm{~K} 9(\mathrm{Ac})$ & 0.61 & 12 & 0.69 & 44 \\
\hline $\mathrm{H} 3 \mathrm{~K} 27 \mathrm{Me}_{3}$ & 0.64 & 40 & 0.68 & 40 \\
\hline $\mathrm{H} 4 \mathrm{Pan}(\mathrm{Ac})$ & 0.67 & 24 & 0.71 & 36 \\
\hline $\mathrm{H} 2 \mathrm{AZ}$ & 0.68 & 28 & 0.72 & 36 \\
\hline 5-Methylcytosine (5MC) & 0.70 & 40 & 0.72 & 40 \\
\hline H2AK119Ub & 0.70 & 36 & 0.78 & 60 \\
\hline $\mathrm{H} 3 \mathrm{~K} \mathrm{Me}_{3}$ & 0.77 & 28 & 0.81 & 28 \\
\hline
\end{tabular}
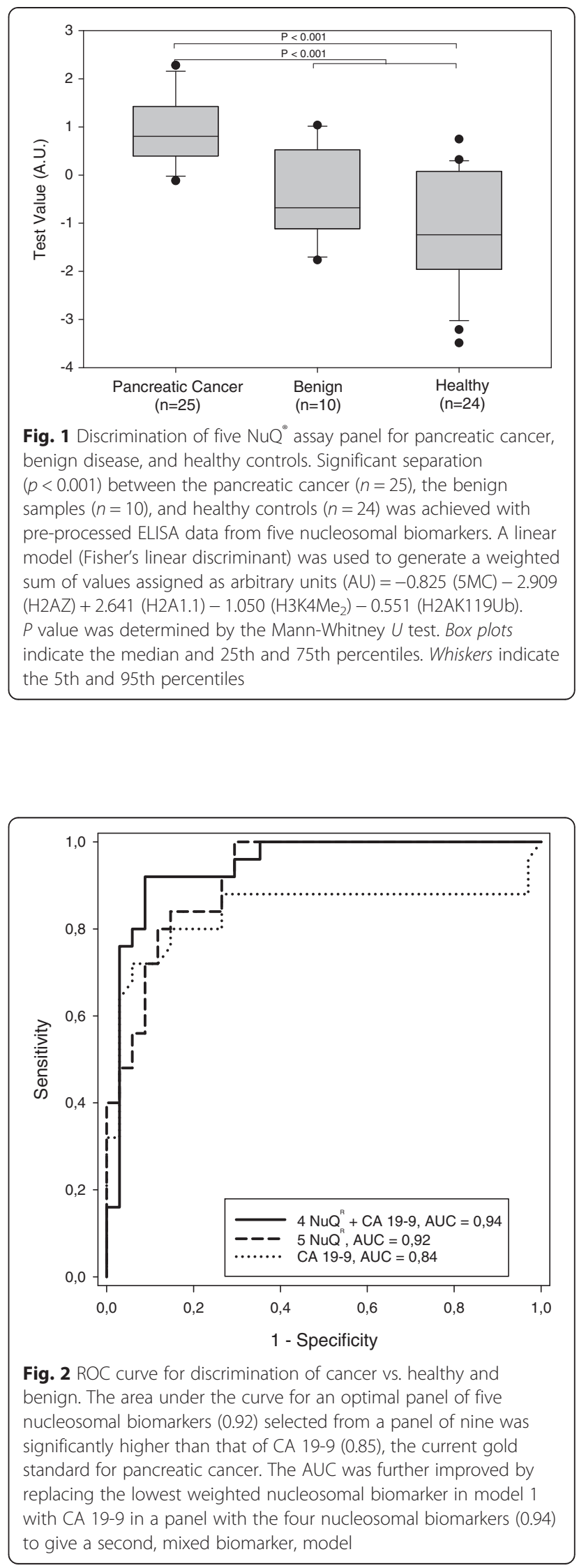


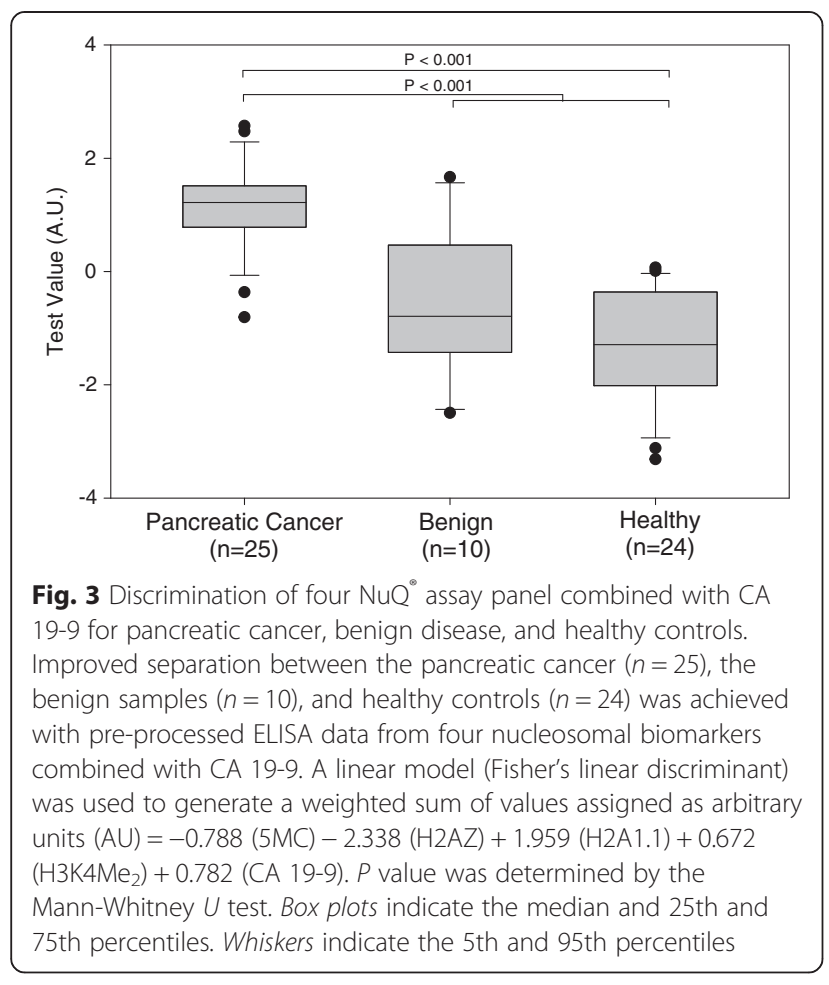

cfnucleosome plus CA 19-9 biomarker panel (model 2) increased the AUC to 0.98 (Fig. 4).

The sensitivities at $90 \%$ specificity for discrimination of cancer vs. healthy and benign as well as cancer vs. healthy groups for the four and five cfnucleosome biomarker panels as well as the four cfnucleosome biomarker panel combined with CA 19-9 increased in line with the AUCs (Table 3).

\section{Discussion}

To our knowledge, this is the first study describing the epigenetic profiling of circulating cfnucleosomes for the detection of pancreatic cancer. Our results suggest that the levels and epigenetic profiles of cfnucleosomes in serum differ in patients with cancer and in control populations. Because epigenetic changes occur early in the neoplastic transformation process, already in preneoplastic stages, cfnucleosome profiles may represent possible biomarkers for the early detection of cancer [21]. Furthermore, the findings that global levels of epigenetic modifications in cfnucleosomes (as opposed to

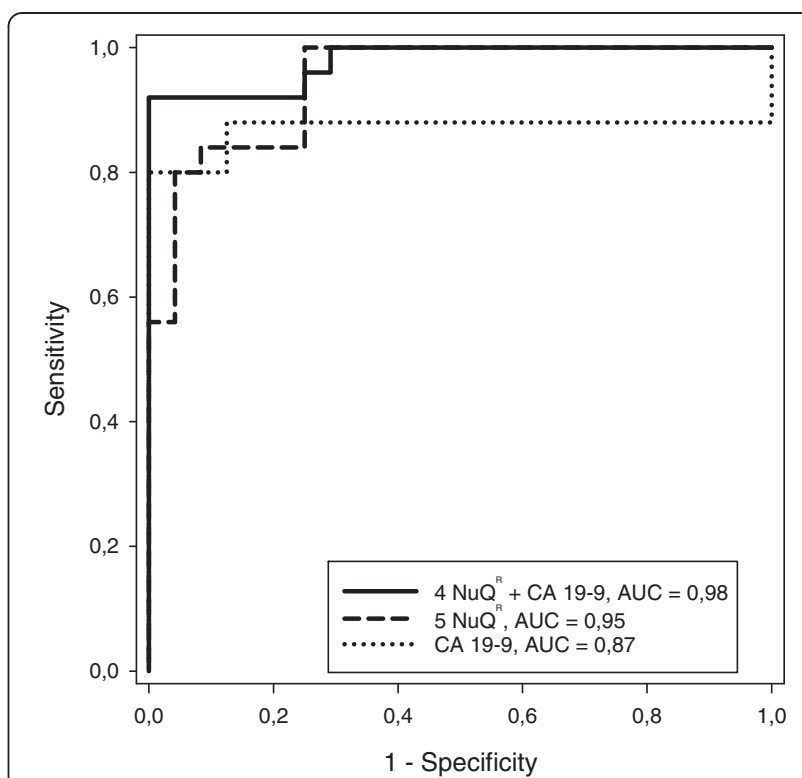

Fig. 4 ROC curve for discrimination of cancer vs. healthy. The area under the curve for an optimal panel of five nucleosomal biomarkers (0.95) selected from a panel of nine was significantly higher than that of CA 19-9 (0.87), the current gold standard for pancreatic cancer. As for discrimination of cancer vs. healthy and benign, the AUC was further improved by replacing the lowest weighted nucleosomal biomarker in model 1 with CA 19-9 in a panel with the four nucleosomal biomarkers (0.98) to give a second, mixed biomarker, model

gene-specific epigenetic profiling) could distinguish pancreatic cancer and benign cases strengthens their ability to be used also in the differential diagnosis and overcomes the previous challenge in separating patients with cancer from benign organ-related diseases. The pancreatic cancer subjects included in this study all had operable stage II disease, and these were detected with high sensitivity.

Despite its current limitations, CA 19-9 is the gold standard to which all new investigational biomarkers are compared. Our data show that while no single cfnucleosome biomarker outperformed CA 19-9 (Additional file 1), these markers can be combined to produce highly clinically sensitive and specific biomarker panels that may also incorporate CA 19-9. A panel of five epigenetic features of cfnucleosomes, identified from an initial screening panel of nine, had a higher diagnostic accuracy than CA 19-9 in serum. The panel of five nucleosomal biomarkers detected

Table 3 Performance of cfnucleosome biomarker panels with and without CA 19-9

\begin{tabular}{|c|c|c|c|c|c|c|c|c|}
\hline \multirow{3}{*}{ Clinical question } & \multicolumn{2}{|c|}{ CA 19-9 } & \multicolumn{2}{|c|}{$4 \mathrm{NuQ}^{\circ}$ assays } & \multicolumn{2}{|c|}{$5 \mathrm{NuQ}^{\oplus}$ assays } & \multicolumn{2}{|c|}{$4 \mathrm{NuQ}^{\circ}$ assays + CA 19-9 } \\
\hline & $\mathrm{AUC}$ & Sensitivity (\%) & $\mathrm{AUC}$ & Sensitivity (\%) & $\mathrm{AUC}$ & Sensitivity (\%) & $\mathrm{AUC}$ & Sensitivity (\%) \\
\hline & & (90 \% specificity) & & (90 \% specificity) & & (90 \% specificity) & & (90 \% specificity) \\
\hline Cancer vs. healthy & 0.87 & 80 & 0.91 & 68 & 0.95 & 84 & 0.98 & 92 \\
\hline Cancer vs. healthy and benign & 0.84 & 72 & 0.90 & 64 & 0.92 & 72 & 0.94 & 92 \\
\hline
\end{tabular}


21 of the 25 pancreatic cancer cases from healthy subjects with two false positive results (sensitivity $84 \%$ at $90 \%$ specificity). Furthermore, the same test was able to distinguish 18 of the pancreatic cancer cases from subjects with other pancreatic diseases or healthy controls with three false positive results (72\% sensitivity at $90 \%$ specificity). There was a single false positive from the healthy group and two in the benign disease group including pre-cancerous intraductal papillary mucinous neoplasms (IPMN). This would represent a potential screening sensitivity for cancer and pre-cancerous disease of $74 \%$.

The markers tested were selected to represent a range of histone isoform, histone modification, and methylated DNA epigenetic signals rather than for suppressive or activating function. The function of epigenetic marks may differ when included at different loci and/or in relation to different genes [8]. Because the present study involves the levels of epigenetic marks on global genome level rather than a gene-specific level, it may be difficult to specify function. In general, we have found that the best discriminating makers in this study have a mixture of functions. 5MC, H2AZ, and H2AK119Ub are thought to be repressive in nature [22], $\mathrm{H} 3 \mathrm{~K}_{4} \mathrm{Me}_{2}$ is thought to be associated with active genes [23], and mH2A1.1 is thought to be associated with cell senescence [24]. From this small sample of markers, it does not appear that one could select discriminating markers on the basis of their activating or suppressive nature.

Inclusion of CA 19-9 in a five-member ELISA panel increased the clinical sensitivity for detection of pancreatic cancer to $92 \%$ at $90 \%$ specificity, both from healthy subjects and from healthy and benign subjects. Only three false positives were detected from the benign group (none from the healthy group), including the same IPMN case identified by the five nucleosomal biomarker panel (model 1).

The main advantage of cfnucleosomes as biomarkers is the rich variety of potential epigenetic features available, which can allow fine-tuning of sensitivity and specificity. Given the large pool of potential epigenetic features present in nucleosomes, it is probable that alternative assays could generate improved panels. A practical advantage of cfnucleosome biomarkers in this respect is that they, like CA 19-9, are ELISA tests that can easily be performed on a single small volume of serum.

When compared to other early detection strategies for pancreatic cancer such as various imaging techniques including endoscopic ultrasound (EUS), circulating cellfree nucleosome assessment offers a potential noninvasive approach to early pancreatic cancer detection.

\section{Conclusions}

In conclusion, our study provides the first evidence of the diagnostic potential of cfnucleosome panels to detect tumor-associated genome-wide epigenetic alterations in serum for the non-invasive detection of pancreatic cancer. Further studies with a broader range of assays in larger patient cohorts are warranted to evaluate the usefulness of these epigenetic markers in diagnosing asymptomatic disease.

\section{Methods}

\section{Serum samples}

Study patients were undergoing treatment at the Department of Surgery, Skåne University Hospital, Lund, Sweden, between March 2012 and June 2014. Blood samples were taken at diagnosis, prior to treatment. Healthy control sera $(n=24)$ were obtained from donors at the local blood donation center.

Serum samples were stored at $-80{ }^{\circ} \mathrm{C}$ in the local biobank until further use. The ethical approval for this study was granted by the institutional review board at Lund University with the approval number 2012/661. All subjects gave written informed consent before taking part in the study. Blood samples were collected in BD SST II Advance tubes (serum separator tubes, $3.5 \mathrm{ml}$, product no. 368498; Becton Dickinson, Franklin Lakes, NJ, USA). The minimum clotting time was $30 \mathrm{~min}$. The samples were centrifuged at $2000 \times g$ at $25^{\circ} \mathrm{C}$ for $10 \mathrm{~min}$, and serum was collected and stored in aliquots at $-80^{\circ} \mathrm{C}$.

\section{cfnucleosome immunoassays}

Nine circulating cfnucleosome structures were measured using NuQ ${ }^{\circ}$ ELISAs (Belgian Volition SA, Namur, Belgium) performed according to the manufacturer's instructions, as reported previously [19]. The assays consist of a single common method and reagent set which employs a nucleosome capture antibody immobilized to the solid phase in conjunction with nine separate detection antibodies directed to bind to the histone modification or variant or DNA modification of interest (mouse monoclonal antibody: anti-H3K4Me $\mathrm{Me}_{2}$ anti-H3K9Ac, anti-H4Pan(Ac), anti-5MC, a rabbit monoclonal antiH2AK119Ub, rabbit polyclonal anti-mH2A1.1, anti$\mathrm{H} 3 \mathrm{~K} 27 \mathrm{Me}_{3}$, anti-H2AZ, anti-H3K9Me $\mathrm{M}_{3}$.

Briefly, serum samples $(10 \mu \mathrm{l}$ in duplicate) were diluted with $50 \mu \mathrm{l} 0.05 \mathrm{M}$ Tris/HCl buffer $\mathrm{pH} 7.5$ and incubated overnight at $4-8{ }^{\circ} \mathrm{C}$ in 96-well microtiter plates coated with a monoclonal anti-nucleosome antibody (Belgian Volition SA, Namur, Belgium). After incubation, wells were washed three times with $200 \mu \mathrm{l}$ of $0.05 \mathrm{M}$ Tris/ $\mathrm{HCl}$ buffer pH 7.5 containing $0.1 \%$ Tween 20 (wash buffer) and $50 \mu \mathrm{l}$ of a biotinylated detection antibody, specific to the epigenetic feature under investigation, was added. Wells were incubated for $90 \mathrm{~min}$ at room temperature and washed three times with $200 \mu \mathrm{l}$ wash buffer, and $50 \mu \mathrm{l}$ of streptavidin-horseradish peroxidase $(\mathrm{HRP}=0.25 \mu \mathrm{g} / \mathrm{ml})$ was added. After incubation for $30 \mathrm{~min}$ at room 
temperature, the wells were washed three times with $200 \mu \mathrm{l}$ of wash buffer, and a peroxidase substrate-2,2'azino-bis(3-ethylbenzothiazoline-6-sulfonic acid)-was added. The optical densities of wells were read after 20 min with an X-Mark Microplate spectrophotometer (BioRad).

Mean imprecision of sample duplicates in the nucleosome assays in this study ranged from 2 to $4 \%$. Intraand inter-plate imprecision for control samples was $<4$ and $<6 \%$, respectively. In larger (27 plate) reproducibility studies (data not shown), intra- and inter-plate reproducibility for nucleosome-associated 5-methylcytosine analyses were 3 and $11 \%$, respectively. For nucleosomeassociated $\mathrm{H} 3 \mathrm{~K} 9 \mathrm{Me} 3$ analyses, intra- and inter-plate reproducibilities were 4 and $11 \%$, respectively.

\section{Statistical analysis}

Samples were assigned to three groups, healthy, cancer, or benign. The data was pre-processed, taking the logarithm to base 2 and dividing by the standard deviation for each assay. Linear models were calculated using (1) logistic regression (LR) and (2) Fisher's linear discriminant (LDA) [20].

These determined the weighted sum of the $\mathrm{NuQ}^{\circ}$ variables, assigned as arbitrary units (AU), that provided optimal discrimination between the cancer and combined healthy and benign groups, identified as the optimal clinical question (Fig. 5). A combination of nine individual $\mathrm{NuQ}^{\circ}$ assays was included for selection (together

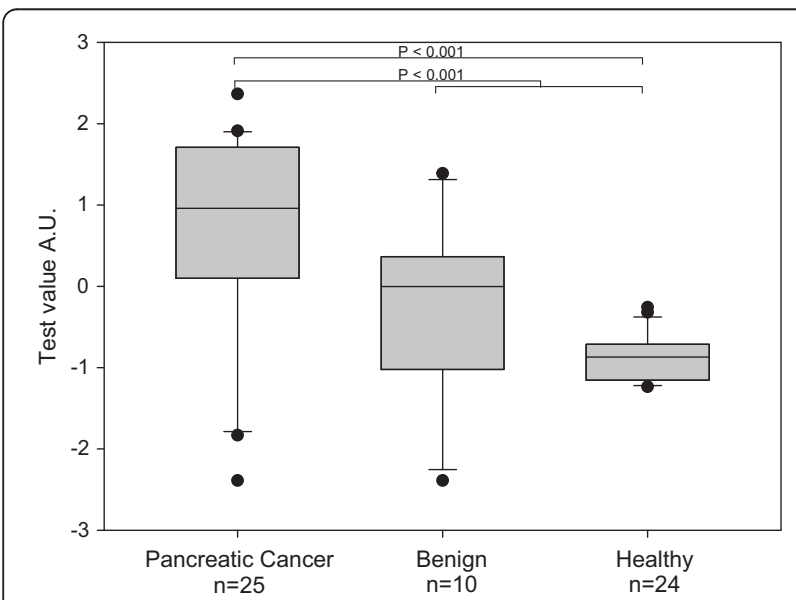

Fig. 5 Discrimination of CA 19-9 for pancreatic cancer, benign disease, and healthy controls. No significant separation between the pancreatic cancer $(n=25)$, the benign samples $(n=10)$, and healthy controls $(n=24)$ was achieved. The performance of CA 19-9 alone is relatively poor with a large degree of overlap between cancer and healthy, cancer as well as benign and healthy emphasizing the poor suitability for CA 19-9 as a biomarker in a screening setting. $P$ value was determined by Mann-Whitney $U$ test. Box plots indicate the median and 25th and 75th percentiles. Whiskers indicate the 5th and 95th percentiles with or excluding CA 19-9 as a potential variable). Models with one to five variables were ranked by area under the receiver operator characteristic curve (AUC). An upper limit of five variables was imposed to avoid overtraining. Equivalent ROC curves were obtained from LR and LDA. The analysis was conducted using the statistical programming language $\mathrm{R}[25,26]$.

\section{Additional file}

Additional file 1: Performance of individual nucleosome assays. ROC curves for each nucleosome assay in cancer vs. healthy or benign and cancer vs. healthy groups.

\section{Competing interests}

DP, MH, ME and JM are employed by Belgian Volition SA, Belgium. The remaining authors have no conflict of interest to declare.

\section{Authors' contributions}

MB drafted the manuscript and participated in the analysis and interpretation of data. DP and MH conducted the nucleosome assays and performed the statistical analyses. DA helped draft the manuscript and took part in the study design and collection of clinical data. ME and JM participated in the design, writing, and review of the manuscript. BA coordinated the local biobank and revised the manuscript for intellectual content. RA conceived of the study and participated in its design and coordination. All authors read and approved the final manuscript.

\section{Authors' information}

Not applicable.

\section{Acknowledgements}

The authors would like to acknowledge Tom Bygott for expertise in bioinformatics. We also appreciate the assistance of Katarzyna Said Hilmersson for collecting the serum samples and organizing the biobank.

\section{Author details}

${ }^{1}$ Department of Surgery, Clinical Sciences, Lund, Lund University and Skåne University Hospital, Lund, SE-221 85 Lund, Sweden. ${ }^{2}$ Belgian Volition SA-Centre Technologique, Rue du Séminaire 20A BE-5000Namur, Belgium.

Received: 11 May 2015 Accepted: 21 September 2015

Published online: 07 October 2015

\section{References}

1. Siegel R, Ma J, Zou Z, Jemal A. Cancer statistics, 2014. CA Cancer J Clin. 2014;64(1):9-29. doi:10.3322/caac.21208.

2. Ghatnekar O, Andersson R, Svensson M, Persson U, Ringdahl U, Zeilon P, et al. Modelling the benefits of early diagnosis of pancreatic cancer using a biomarker signature. Int J Cancer. 2013;133(10):2392-7. doi:10.1002/ijc.28256.

3. Goonetilleke KS, Siriwardena AK. Systematic review of carbohydrate antigen (CA 19-9) as a biochemical marker in the diagnosis of pancreatic cancer. Eur J Surg Oncol. 2007;33(3):266-70. doi:10.1016/j.ejso.2006.10.004.

4. Duffy MJ, Sturgeon C, Lamerz R, Haglund C, Holubec VL, Klapdor R, et al. Tumor markers in pancreatic cancer: a European Group on Tumor Markers (EGTM) status report. Ann Oncol. 2010;21(3):441-7. doi:10.1093/annonc/ mdp332.

5. Omura N, Goggins M. Epigenetics and epigenetic alterations in pancreatic cancer. Int J Clin Exp Pathol. 2009;2(4):310-26.

6. Simmons D. Epigenetic influences and disease. Nature Education. 2008;1(1):6.

7. Neureiter D, Jager T, Ocker M, Kiesslich T. Epigenetics and pancreatic cancer: pathophysiology and novel treatment aspects. World J Gastroenterol. 2014;20(24):7830-48. doi:10.3748/wjg.v20.i24.7830.

8. Fullgrabe J, Kavanagh E, Joseph B. Histone onco-modifications. Oncogene. 2011;30(31):3391-403. doi:10.1038/onc.2011.121. 
9. Bianco-Miotto T, Chiam K, Buchanan G, Jindal S, Day TK, Thomas M, et al. Global levels of specific histone modifications and an epigenetic gene signature predict prostate cancer progression and development. Cancer Epidemiol Biomarkers Prev. 2010;19(10):2611-22. doi:10.1158/1055-9965.EPI-10-0555.

10. Manuyakorn A, Paulus R, Farrell J, Dawson NA, Tze S, Cheung-Lau G, et al. Cellular histone modification patterns predict prognosis and treatment response in resectable pancreatic adenocarcinoma: results from RTOG 9704. J Clin Oncol. 2010;28(8):1358-65. doi:10.1200/JCO.2009.24.5639.

11. Wei Y, Xia W, Zhang Z, Liu J, Wang H, Adsay NV, et al. Loss of trimethylation at lysine 27 of histone $\mathrm{H} 3$ is a predictor of poor outcome in breast, ovarian, and pancreatic cancers. Mol Carcinog. 2008;47(9):701-6. doi:10.1002/mc.20413.

12. Jahr S, Hentze H, Englisch S, Hardt D, Fackelmayer FO, Hesch RD, et al. DNA fragments in the blood plasma of cancer patients: quantitations and evidence for their origin from apoptotic and necrotic cells. Cancer Res. 2001;61(4):1659-65.

13. Mouliere F, Rosenfeld N. Circulating tumor-derived DNA is shorter than somatic DNA in plasma. Proc Natl Acad Sci U S A. 2015;112(11):3178-9. doi:10.1073/pnas.1501321112.

14. Kremer A, Wilkowski R, Holdenrieder S, Nagel D, Stieber P, Seidel D. Nucleosomes in pancreatic cancer patients during radiochemotherapy. Tumour Biol. 2005;26(1):44-9. doi:10.1159/000084339.

15. Wittwer $C$, Boeck $S$, Heinemann V, Haas M, Stieber P, Nagel D, et al. Circulating nucleosomes and immunogenic cell death markers HMGB1, sRAGE and DNAse in patients with advanced pancreatic cancer undergoing chemotherapy. Int J Cancer. 2013;133(11):2619-30. doi:10.1002/ijc.28294.

16. Toiyama Y, Okugawa Y, Goel A. DNA methylation and microRNA biomarkers for noninvasive detection of gastric and colorectal cancer. Biochem Biophys Res Commun. 2014;455(1-2):43-57. doi:10.1016/j.bbrc.2014.08.001.

17. Newman AM, Bratman SV, To J, Wynne JF, Eclov NC, Modlin LA, et al. An ultrasensitive method for quantitating circulating tumor DNA with broad patient coverage. Nat Med. 2014;20(5):548-54. doi:10.1038/nm.3519.

18. Holdenrieder S, Stieber $P$, Bodenmuller H, Busch M, Fertig G, Furst $H$, et al. Nucleosomes in serum of patients with benign and malignant diseases. Int J Cancer. 2001;95(2):114-20.

19. Holdenrieder S, Dharuman Y, Standop J, Trimpop N, Herzog M, Hettwer K, et al. Novel serum nucleosomics biomarkers for the detection of colorectal cancer. Anticancer Res. 2014;34(5):2357-62.

20. Pohar M, Blas M, Turk S. Comparison of logistic regression and linear discriminant analysis: a simulation study. Metodološki zvezki. 2004;1(1):143-61.

21. Kovalchuk O, Tryndyak VP, Montgomery B, Boyko A, Kutanzi K, Zemp F, et al. Estrogen-induced rat breast carcinogenesis is characterized by alterations in DNA methylation, histone modifications and aberrant microRNA expression. Cell Cycle. 2007;6(16):2010-8.

22. Biran A, Meshorer E. Concise review: chromatin and genome organization in reprogramming. Stem Cells. 2012;30(9):1793-9. doi:10.1002/stem.1169.

23. Sims 3rd RJ, Nishioka K, Reinberg D. Histone lysine methylation: a signature for chromatin function. Trends Genet. 2003;19(11):629-39. doi:10.1016/ j.tig.2003.09.007.

24. Sporn JC, Jung B. Differential regulation and predictive potential of MacroH2A1 isoforms in colon cancer. Am J Pathol. 2012;180(6):2516-26. doi:10.1016/j.ajpath.2012.02.027

25. R Core Team. A language and environment for statistical computing Vienna, Austria: R Foundation for Statistical Computing; 2013. http://www.R-project.org/.

26. Sing T, Sander O, Beerenwinkel N, Lengauer T. ROCR: visualizing classifier performance in R. Bioinformatics. 2005;21(20):3940-1. doi:10.1093/bioinformatics/bti623.

\section{Submit your next manuscript to BioMed Central and take full advantage of:}

- Convenient online submission

- Thorough peer review

- No space constraints or color figure charges

- Immediate publication on acceptance

- Inclusion in PubMed, CAS, Scopus and Google Scholar

- Research which is freely available for redistribution

Submit your manuscript at www.biomedcentral.com/submit 